
\section{Did COPD}

Although chronic obstructive pulmonary disease (COPD) is a very common respiratory disorder, few interventions have been shown to affect mortality from the disease. Inhaled corticosteroids have been found to reduce COPD exacerbations and improve health status and, as exacerbations have an effect on disease progression, one would expect inhaled corticosteroids to reduce mortality. However, studies of inhaled steroids in COPD have not been powered to investigate mortality. In this month's issue of Thorax we report a meta-analysis by Sin and colleagues which shows, for the first time, that inhaled corticosteroids reduce all-cause mortality in COPD. This paper is important because it resulted from a unique collaboration between academia and industry that allowed a pooled analysis of the original data from seven trials to obtain a large dataset of 5085 patients. As in any analyses of this nature there are limitations, but these are addressed in the paper and accompanying editorial. A number of questions about the use of inhaled corticosteroids will still need to be answered but, in the coming year, we should have the results of the first adequately powered study of the effects of inhaled corticosteroids on mortality in COPD.

See pages 977 and 992

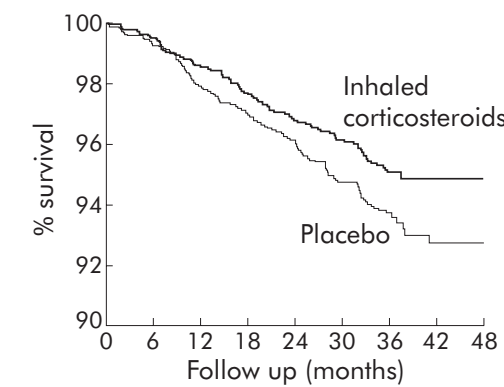

Number $5085441034293023295128932331 \quad 867 \quad 22$ of study

Comparison of Kaplan-Meier survival curves between patients treated with inhaled corticosteroids and placebo in COPD (log rank $p=0.039$ )

\section{PREMATURITY AND RSV}

Respiratory syncytial virus (RSV) is an important cause of morbidity in childhood. In this issue of Thorax Broughton and colleagues describe a follow up study in prematurely born infants (born before 32 weeks of gestational age) to determine the impact of RSV infection, both in hospital and in the community, on respiratory morbidity and healthcare utilisation. At follow up to a corrected age of 1 year, infants who suffered RSV infection had more hospital admissions, days in hospital, and more cough and wheeze. Risk factors for RSV infection included the number of siblings and maternal smoking in pregnancy. An important result of this study was that RSV infections in the community, as well as those needing admission to hospital, were associated with respiratory morbidity at follow up.

See page 1039

\section{PROMISE FOR TNF $\alpha$ IN SEVERE ASTHMA}

$\mathrm{TNF} \alpha$ is a major therapeutic target in a number of chronic inflammatory disorders that are characterised by a Thl type immune response. Although asthma is considered to be a Th2 type disorder, severe asthma also has features of a Thl type inflammatory response. In this month's Thorax, Howarth and colleagues report studies on the role of TNF $\alpha$ in symptomatic corticosteroid dependent asthma. TNF $\alpha$ levels in bronchoalveolar lavage fluid from severe asthmatics were higher than in samples from mild asthmatics or healthy controls. An open label study was performed with an anti-TNF $\alpha$ intervention (etanercept) and this showed improvement in asthma symptoms, lung function, and bronchial hyperresponsiveness. This is the first study to evaluate TNF $\alpha$ blockade in asthma and, as the authors conclude, large controlled studies are needed in severe asthma which is still one of the most important unmet clinical needs.

See page 1012

\section{SURVIVAL IN PAH}

Idiopathic pulmonary arterial hypertension (PAH), formerly known as primary pulmonary hypertension, is associated with considerable morbidity and mortality. New mechanisms for PAH have led to new treatments and, in this issue of Thorax, we publish a paper by Sitbon and colleagues on improving survival in this condition. The dual endothelin receptor antagonist bosentan is the first oral treatment approved for PAH. Survival in patients treated with bosentan was compared with historical data from patients treated with intravenous epoprostenol. There was no evidence that the bosentan group had a worse long term outcome than the epoprostenol group. As Mehta and Shoemaker conclude in their editorial, these new treatments will also be used by patients with other types of pulmonary hypertension.

See pages 980 and 1025

\section{AND NOW AT THE END OF THE YEAR!}

Another year passes and I would like to take the opportunity to thank all our readers and authors for their support of the journal over the past year. Please continue to send us your very best original papers in the coming year 2006. Special thanks are due to our reviewers, and on pages 989-91 we publish the names of all the reviewers who have helped us over the past 12 months. This issue also contains the Annual Report and you will notice just how busy Thorax has been; the impact factor has increased to 5.040 and we have instituted a number of innovations. The most important objective of the journal is that all members of the global respiratory community can find something each month of interest to them in Thorax.

See page 987 